\title{
Gangstörungen bei psychiatrischen Erkrankungen
}

\author{
Maria Perfahl, Albert Putzhammer \\ Klinik und Poliklinik für Psychiatrie, Psychosomatik und Psychotherapie \\ der Universität Regensburg \\ (Direktor: Prof. Dr. H. E. Klein)
}

psychoneuro 2005; 31 (7+8): 374-378

Gangstörungen sind ein häufiges Symptom psychiatrischer Erkrankungen. Sie können zu erheblichen Einschränkungen der Mobilität führen und haben eine immense subjektive und sozioökonomische Bedeutung für den Betroffenen. Der stabile und flüssige aufrechte Gang des Menschen ist ein komplexer automatisierter Bewegungsablauf, der das störungsfreie Zusammenwirken zahlreicher Gehirnfunktionen voraussetzt. Zur objektiven Untersuchung des Gangs werden die Videografie, das Verfahren zur Schrittanalyse nach Brinkmann sowie Ultraschalloder Infrarot-Bewegungsanalysesysteme eingesetzt. Sie erlauben eine genauere Analyse von Gangstörungen. Besondere Bedeutung in der Psychiatrie haben das Erkennen von psychogenen Gangstörungen, Gangstörungen im Rahmen von affektiven Erkrankungen sowie Gangstörungen bei Erkrankungen aus dem schizophrenen Formenkreis. Bei Letzteren ist die Differenzierung zwischen primären Krankheitssymptomen und extrapyramidal-motorischen Nebenwirkungen der Neuroleptika besonders wichtig.

W arum sollte sich ein Psychiater oder ein Nervenarzt mit Gangstörungen beschäftigen? Hört man von Veränderungen motorischer Funktionen im Rahmen psychiatrischer Erkrankungen, dann denkt man meist an Veränderungen der Mimik, die einen Einblick in die Affektlage der Patienten ermöglichen, an Veränderungen der allgemeinen motorischen Aktivität, wie z.B. bei stuporösen Zuständen, und vielleicht noch an Störungen der Handfunktion, wie den Tremor bei psychomotorischer Erregung. An Störungen des Gangs denkt man in der Regel erst in zweiter Linie, wohl auch deshalb, weil psychiatrische Konsultationen meist im Sitzen stattfinden und Gangstörungen wegen ihrer Komplexität oftmals nur schwer exakt zu beschreiben sind. Ähnlich wie die Sprache ist jedoch der aufrechte zweibeinige Gang eine cha- rakteristische Ausdrucksform der komplexen Leistungsfähigkeit des menschlichen Gehirns. Deshalb sollten Störungen des Gehens unbedingt auch bei psychiatrischen $\mathrm{Er}$ krankungen Beachtung finden. Ein weiterer wichtiger Grund sich mit Gangstörungen bei psychiatrischen Patienten zu beschäftigen, ist die immense subjektive und sozioökonomische Bedeutung einer Einschränkung der Mobilität. Störungen des Gangs führen - da sie für jedermann erkennbar sind - zu Stigmatisierung und sozialer Isolation und sie können erhebliche Einschränkungen der Erwerbsfähigkeit verursachen.

\section{Physiologie des Gangs}

Der stabile aufrechte Gang des Menschen ist neben der Fähigkeit zum Spracherwerb eine komplexe Leistung des ZNS, die ihn eindeutig von allen Tieren unterscheidet. Man

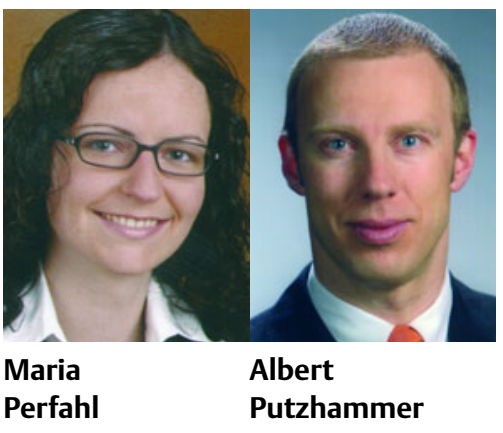

Perfahl kann den menschlichen Gang als eine zyklische Vorwärtsbewegung eines vielgelenkigen Systems im dreidimensionalen Raum definieren, bei der wechselseitig zwei Beine zur Vorwärtsbewegung und zur jeweiligen Unterstützung eingesetzt werden und von denen mindestens eines Bodenkontakt hat (13). Als Gangzyklus werden dabei die Vorgänge bezeichnet, die während des Gehens zwischen zwei aufeinander folgenden gleichen Bewegungsereignissen derselben Extremitätenseite ablaufen. Meist wird der erste Bodenkontakt des rechten Fußes als Beginn eines Gangzyklus gewählt. Er endet dann mit dem folgenden Bodenkontakt des rechten Fußes (3). Der Gangzyklus beschreibt damit also nichts anderes als zwei aufeinander folgende Schritte und gliedert sich zeitlich in zwei Doppelstandphasen, während derer beide Beine Kontakt zum Boden haben, sowie eine rechte und eine linke einbeinige Stand- und Schwungphase. Der Anteil der Standphase eines jeden Beins an der Zykluszeit beträgt etwa $60 \%$, der der Schwungphase ungefähr 40\%. Im Erwachsenenalter beträgt die Doppelstandphase (DS) maximal 13\% der Zyklusdauer. Abbildung 1 stellt diese Unterteilungen des Gangzyklus grafisch dar.

Der Abstand zwischen dem Bodenkontakt des rechten Beines und dem des linken Beines ist die rechte Schrittlänge. Die Frequenz der Ab- 


\section{Abb. 1 Unterteilungen des Gangzyklus}

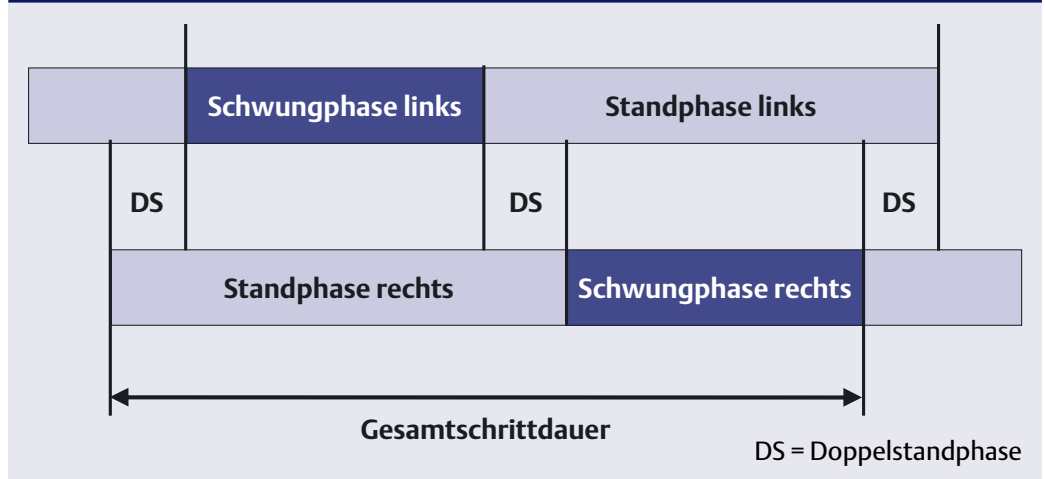

Abb. 2 Gehgeschwindigkeit

Geschwindigkeit = Schrittlänge $\mathrm{x}$ Kadenz

folge von linken und rechten Schritten, also die Anzahl der Schritte pro Minute, bezeichnet man als Kadenz. Die Gehgeschwindigkeit ist das Produkt aus Schrittlänge und Kadenz (Abb. 2). Sie kann bei gesunden Personen zunächst durch eine Veränderung der Schrittlänge und in der Folge auch durch eine Regulation der Kadenz erhöht oder erniedrigt werden. Für eine Steigerung der Gehgeschwindigkeit wird die Schrittlänge vergrößert und die Kadenz erhöht. Dabei nehmen die Dauer der Schwung- und der Standphase ab $(4,12)$. Langsames Gehen wird durch eine Verringerung der Schrittlänge und eine niedrigere Kadenz erreicht. Zahlreiche „Stellgrößen“ tragen zur Regulation dieser Parameter bei (Abb. 3). Sie werden im Regelfall der Situation angepasst und so eingestellt, dass eine optimale Ressourcennutzung gewährleistet ist, also die ideale Gehgeschwindigkeit mit dem geringsten Energieaufwand erreicht wird. Da während des Gehens der Körperschwerpunkt in der Abfolge der Schritte ständig auf und ab sowie von links nach rechts und zurück bewegt wird, wird der gesamte Energieaufwand dann am geringsten, wenn diese Auslenkungen möglichst gering gehalten werden. Ein unsicherer breitbeiniger Gang oder eine übermäßige Auf- und Abbewegung des Körpers beim Gehen führen zu einem erhöhten Energieverbrauch. der so genannten Chronofotografie Bewegungsaufnahmen mit zeitlich gekoppelten Fotoapparaten machte (Abb. 5). Dabei erreichte er eine Auflösung von 25 Bildern pro Minute. Er konnte so erstmals den charakteristischen Ablauf eines Gangzyklus in seinen Einzelheiten für das menschliche Auge fassbar machen. Viele seiner Bilder sind in einem Internetauftritt der Cinémathèque francaise $\mathrm{zu}$ sehen (www.expo-marey.com).

Eine Weiterentwicklung dieser Methodik ist die Videografie, bei der mit einer oder mehreren Kameras das Gangbild eines Patienten oder Probanden aufgezeichnet wird. Danach werden die Aufzeichnungen in Einzelbildern analysiert und die Gangparameter quantifiziert. Nachteilig an dieser Methode ist, dass trotz Festlegung von Auswertekriterien betrachterabhängige Fehler auftreten können. Deshalb sollte die Auswertung bei diesem Verfahren immer durch zwei unabhängige Personen erfolgen.

Ein weiteres häufig verwendetes Analysesystem ist das Verfahren zur Schrittanalyse nach Brinkmann. Dabei werden die Schrittparameter anhand von Fußspuren auf einer Gangbahn und in Verbindung mit einem elektronischen Lichtschranken- und Stoppuhrsystem ermittelt. Eine Kombination mit der Videografie erlaubt dann die objektive Erfassung der wichtigsten Gangparameter.

\section{Abb. 3 Die Gangterminanten und ihre Funktion}

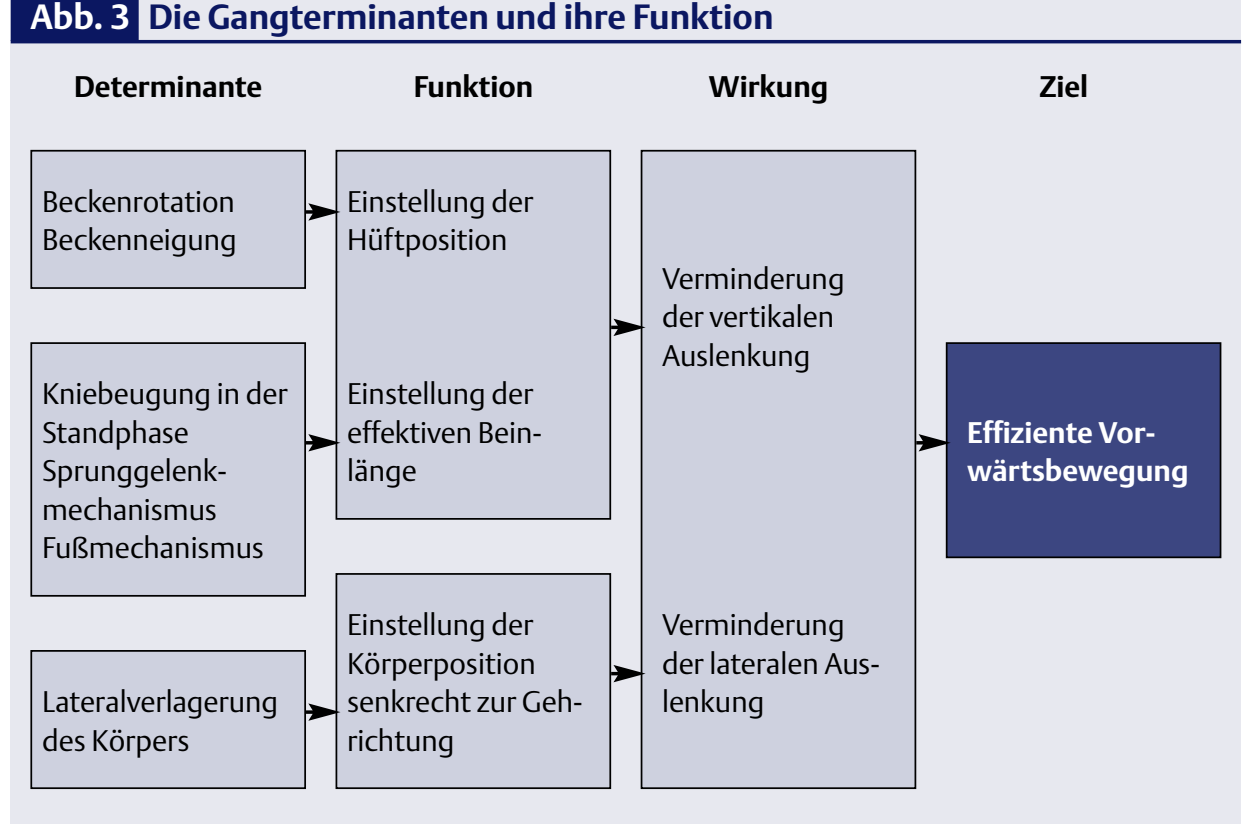




\section{Abb. 4 Etienne-Jules Marey}

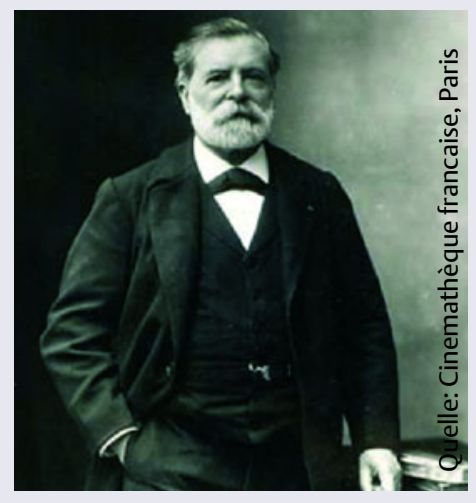

Etienne-Jules Marey, 1830-1904, führte die ersten methodischen Analysen durch

Mittlerweile sind durch den Einsatz von Ultraschall- oder InfrarotBewegungsanalysesystemen dreidimensionale kinematische Untersuchungen des menschlichen Gangs möglich. Dazu werden Positionsmarker, z.B. Ultraschallsender oder Infrarotmarker, an relevanten Körperstellen angebracht. Ihre Position im Raum kann dann durch die Messsysteme berechnet werden. Ultraschallmesssysteme basieren auf der Messung von Schalllaufzeiten. Die an definierten gelenknahen Körperstellen angebrachten kleinen Ultraschallsender senden mit hoher Rate Impulse aus, die von fest montierten Ultraschallmikrofonen aufgenommen werden. Aus der Differenz der Schalllaufzeiten zu den einzelnen Mikrofonen errechnet das System die Position der einzelnen Sender. Die zeitliche Auflösung der Systeme liegt bei ca. 10 Millisekunden, die räumliche Auflösung deutlich unter einem Millimeter. Aus der Abfolge der errechneten Positionen wird die Bewegung der Sender digitalisiert nachvollzogen. So können zahlreiche Kennparameter von Bewegungen bestimmt werden. Bei der Analyse des Gangs sind dies z.B. Gehgeschwindigkeit, Schrittlänge, Kadenz und zahlreiche Gelenkwinkel. Das Prinzip der Messung ist in Abbildung 6 dargestellt. Diese Methode ist in der klinischen Forschung - vor allem in Neurologie, Orthopädie und Sportmedizin - mittlerweile weit verbreitet und wird auch in der Psychiatrie eingesetzt. Gründe dafür sind auch, dass die Anwendung sehr variabel ist und die Analyse einer großen Anzahl von Bewegungsabläufen, von der Mimik über Handbewegungen bis zum Gehen, erlaubt. Für den routinemäßigen Einsatz in der Klinik sind solche Verfahren jedoch meist zu aufwändig. Dazu kommt, dass sie noch zu wenig standardisiert sind und in einigen Bereichen Belege dafür fehlen, dass die Verfahren sensitiv und spezifisch genug einzelne Krankheitsbilder voneinander unterscheiden. Somit stützt sich gerade die klinische Beobachtung und Einordnung von Gangstörungen weiterhin vor allem auf subjektive Eindrücke und rein deskriptive Beschreibungen.

\section{Psychogene Gangstörungen}

Psychogene Gangstörungen können im Rahmen von Konversionsstörungen und Somatisierungsstörungen auftreten. Sie gehören zu den Erkrankungen, deren Diagnose am schwierigsten und häufig umstritten ist. Meist erfolgt die Diagnose einer psychogenen Gangstörung erst nach - teilweise wie-

\section{Abb. 5 Chronofotografie}

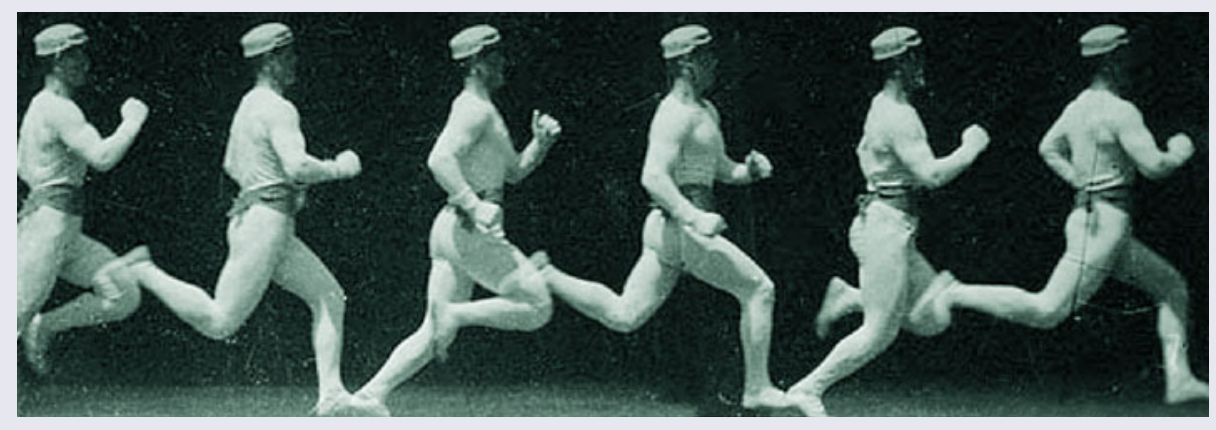

Bewegungsaufnahmen mit zeitlich gekoppelten Fotoapparaten

derholtem - Ausschluss einer zugrundeliegenden neurologischen oder orthopädischen Erkrankung (2). Dabei ist es für den Patienten, der keine körperliche Erkrankung hat oder dessen körperliche Erkrankung nicht sein momentanes Problem begründet, sehr belastend in eine endlose Schleife von Untersuchungen und nicht angemessenen Behandlungsversuchen zu geraten. Psychogene Gangstörungen treten häufiger bei jüngeren Patienten auf (10). Sie sind häufiger bei Frauen als bei Männern. Hat man bei einem Patienten den Verdacht auf eine psychogene Ursache einer Gangstörung, ist es besonders wichtig eine ausführliche psychiatrische Anamnese zu erheben. Ein relativ typisches Zeichen einer psychogenen Verursachung ist das besonders bunte Bild von Symptomen. So kann zu der Gangstörung eine lange Liste weiterer Beschwerden kommen. Dabei kann jede einzelne einer spezifischen Erkrankung zuordenbar sein. In der Summe jedoch können sie nicht das Resultat einer Erkrankung sein. Meist gibt es auch eine große Diskrepanz zwischen den geschilderten Beschwerden und der Beeinträchtigung im täglichen Leben. Besonders aufschlussreich kann demzufolge die Erhebung der Fremdanamnese bei Familienangehörigen und Freunden sein. Psychogene Gangstörungen beginnen meist abrupt und enden in manchen Fällen dann auch genauso plötzlich wieder. Oft variiert die Ausprägung der Symptome stark, was für die meisten somatischen Erkrankungen untypisch ist. Liegt bei einem Patienten eine psychogene Gangstörung vor, zeigt er in der Regel einen auffallend langsamen Gang, der mit erhöhter Anstrengung und schneller Ermüdbarkeit einhergeht. Kommt es beispielsweise im Rahmen einer histrionischen Persönlichkeitsstörung zu einer dissoziativen Gangstörung, ist der Gang des Patienten meist bizarr und verschlechtert sich unter Beobachtung. Zugrunde liegt häufig die unbewusste Flucht vor einer als außergewöhnlich erlebten inneren oder äußeren Konfliktsituation. Besonders bizarr sind psychogene Gangstörungen, bei denen es dem 
Patienten trotz normaler Beinfunktionen in Rückenlage nicht möglich ist zu stehen (Astasie) oder zu gehen (Abasie).

\section{Gangstörungen bei depressiven Patienten}

Die Motorik eines Menschen wird durch seine Gefühle stark beeinflusst. Störungen der Motorik werden deshalb seit langem als ein Symptom depressiver Episoden verstanden. Kraepelin beschrieb 1896 depressive Menschen wie folgt: „Die Körperhaltung zeigt den Mangel an Spannkraft, das schlaffe Bedürfnis nach möglichster Ruhe und stabilem Gleichgewichte, der Kopf ist gesenkt, der Rücken gebeugt und der ganze Körper nach dem Gesetze der Schwere in sich zusammengesunken. Die Sprache ist gewöhnlich leise und zögernd, die Bewegungen geschehen langsam und ohne Kraft. Bisweilen bildet sich die lähmende Willenlosigkeit zu einer förmlichen Bettsucht aus.“ Ähnliches schrieb Bleuler im Jahr 1916: „Die Bewegungen werden mühsam, langsam, kraftlos. Die Glieder sind schwer „wie Blei“. Bewegungen kosten ebensoviel Anstrengung wie das Denken." Das macht deutlich, dass Veränderungen der Motorik bekannte klinisch beobachtbare Zeichen depressiver Erkrankungen sind. Sie gehören sogar zu den Kriterien für affektive Störungen in aktuellen Klassifikationssystemen wie der ICD-10 oder dem DSM-IV. Die Abnahme der generellen motorischen Aktivität korreliert mit der Schwere der vom Patienten erlebten depressiven Symptome (5). Der Gang depressiver Patienten wurde zunächst von Sloman et al. (1982) mit Hilfe der Videografie untersucht. Dabei konnte gezeigt werden, dass der Gang gesunder Kontrollpersonen überwiegend propulsiv, der depressiver Patienten überwiegend hebend (lifting motion) ist (11). Bei den depressiven Patienten war die Schrittlänge gegenüber der Kontrollgruppe signifikant verkürzt. Es bestand eine enge Beziehung zwischen der Stimmung und den erhobenen Gangparametern. Räumliche und zeitliche Parameter des Gangs bei depressiven Patienten wurden vor allem von der Ar- beitsgruppe Lemke et al. ausführlich untersucht. Sie verwendeten dazu eine mit einem transparenten Zeichenpapier ausgelegte Gehstrecke, auf der die Probanden in selbstgewählter Geschwindigkeit gingen. Die räumlichen Gangparameter wurden mit Hilfe von Kupferblechplatten, die an der Unterseite der Schuhe der Probanden angebracht wurden und sich in dem Papier abdrückten, bestimmt. Zur Bestimmung der zeitlichen Parameter wurden ein System von Infrarot-Lichtbarrieren und die Aufzeichnung durch ein Videosystem verwendet. Die depressiven Patienten hatten im Vergleich zu den gesunden Kontrollpersonen eine signifikant verminderte Gehgeschwindigkeit in Verbindung mit einer Schritthypometrie und einer verlängerten Gangzyklusdauer. Die verlängerte Zyklusdauer ergab sich aus einer Verlängerung der Standphasen und der Doppelstandphase. Im Hinblick auf die Regulation der Gehgeschwindigkeit bestand bei den depressiven Patienten eine signifikante Korrelation von Geschwindigkeit und Kadenz, die bei gesunden Kontrollpersonen nicht nachweisbar ist. Da dies auch bei Patienten mit Parkinson-Erkrankung gefunden werden konnte, ist diese Korrelation zwischen Geschwindigkeit und $\mathrm{Ka}$ denz als Hinweis auf eine Beteiligung der Basalganglien in der Pathogenese der Gangstörungen depressiver $\mathrm{Pa}$ tienten zu werten (6).

\section{Gangstörungen schizophren erkrankter Patienten}

Besonderes Interesse sollte auch Gangstörungen bei Patienten mit Psychosen aus dem schizophrenen Formenkreis gelten. Meist denkt man bei psychomotorischen Störungen im Rahmen einer Schizophrenie zunächst an die extrapyramidal-motorischen Nebenwirkungen der zur Behandlung eingesetzten Neuroleptika. Psychomotorische Störungen bei Patienten mit Schizophrenien wurden jedoch lange vor der Einführung der Neuroleptika beschrieben und von Kraepelin und Bleuler sogar als Kardinalsymptome der Schizophrenie betrachtet. Dabei fassten sie Bilder wie Stupor, Stereotypien, Manierismen, Negativismen

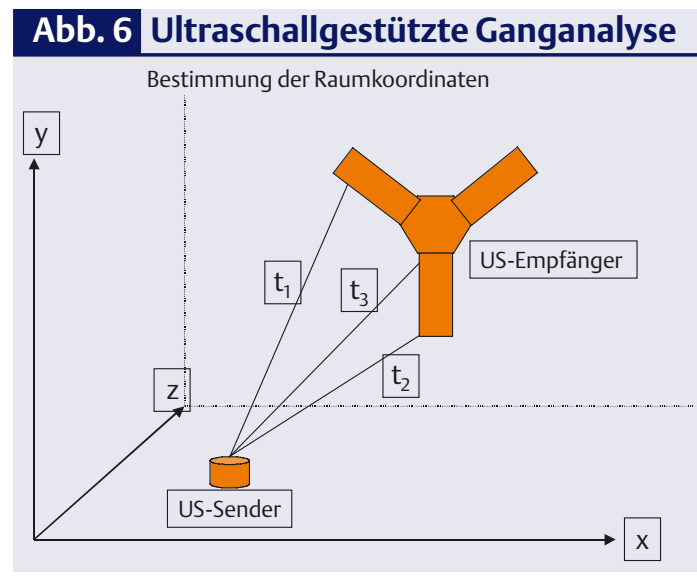

oder Automatien zum katatonen Symptomenspektrum zusammen. Auch die so genannten neurological soft signs (NSS), also diskrete normabweichende motorische und sensorische Leistungen, werden als primäre Krankheitssymptome gewertet. Damit ist es besonders wichtig zu beachten, dass motorische Symptome bei schizophren erkrankten Patienten sowohl durch die Krankheit an sich verursacht, als auch unerwünschte Wirkungen der antipsychotischen Medikation sein können. Da die erheblichen extrapyramidalen Nebenwirkungen, die bei der Therapie vor allem mit den älteren, so genannten konventionellen Neuroleptika auftreten, häufig ein Grund für Non-Compliance und einen Therapieabbruch durch den Patienten sind, ist die ätiologische Einordnung motorischer Störungen als Krankheitssymptom oder als Nebenwirkung eine zentrale Frage in der Behandlung der Schizophrenie. Hier kann vor allem eine genaue Anamnese wertvolle Hinweise geben.

Das Vorliegen von extrapyramidal-motorischen Störungen unter neuroleptischer Therapie wird dabei meist klinisch beschreibend erfasst. Soll deren Ausprägungsgrad näher bestimmt werden, verwendet man in der Regel klinische RatingSkalen, wie z.B. die Extrapyramidal Symptoms Rating Scale (ESRS) von Chouinard (1).

In eigenen Untersuchungen zur Unterscheidung zwischen krankheitsbedingten und pharmakogenen Störungen der Motorik sowie zur Quantifizierung des Ausmaßes der Beeinträchtigungen wurde ein ultra- 
schallgestützes Bewegungsanalysesystem verwendet, wie es bereits oben beschrieben wurde $(7,8,9)$. Bei einer mit Hilfe dieses Systems durchgeführten Untersuchung wurde der freie Gang von unbehandelten schizophren erkrankten Patienten, von mit konventionellen Neuroleptika behandelten Patienten, von mit dem atypischen Neuroleptikum Olanzapin behandelten Patienten und von gesunden Kontrollpersonen verglichen. Es konnte gezeigt werden, dass die frei gewählte Gehgeschwindigkeit bei allen schizophren erkrankten Patienten signifikant niedriger als bei den gesunden Kontrollpersonen war. Innerhalb der Patientengruppen war die Gehgeschwindigkeit bei Patienten unter konventioneller neuroleptischer Medikation signifikant niedriger als bei Patienten unter atypischer neuroleptischer Medikation bzw. als bei unbehandelten Patienten. Diese langsamere Geschwindigkeit wurde nicht durch eine Störung der Schrittfolge (Kadenz) verursacht, sondern war hauptsächlich auf eine signifikant kürzere Schrittlänge zurückzuführen. Es ist also davon auszugehen, dass es bei schizophren erkrankten Patienten bereits aufgrund der Erkrankung zu einer Gangstörung kommt, welche durch die Medikation mit konventionellen Neuroleptika noch weiter verschlechtert wird. Eine Medikation mit atypischen Neuroleptika scheint keine zusätzliche Verschlechterung der motorischen Fähigkeiten zu verursachen. Damit unterstreicht dieses Ergebnis die Vorteile der Behandlung mit atypischen Neuroleptika in Hinblick auf die medikamenteninduzierten Störungen des Gangs gegenüber einer Behandlung mit konventionellen Neuroleptika. Es unterstreicht aber auch die Existenz und die Bedeutung primär krankheitsbedingter Gangstörungen, die nicht Folge der Behandlung mit Neuroleptika sind.

\section{Ausblick}

Der Blick auf Störungen des Gangs bei Patienten mit psychiatrischen Erkrankungen ist klinisch und wissenschaftlich interessant. Gangstörungen treten bei vielen psychiatrischen Erkrankungen auf und sind ein nicht zu unterschätzendes Problem für das subjektive Wohlbefinden und für die sozioökonomische Situation der Patienten. Genaue kinematische Untersuchungen von Gangstörungen ermöglichen tiefere Einblicke in die pathophysiologischen Grundlagen psychischer Erkrankungen und können in Zukunft dazu beitragen, die Differenzierung von somatischen Grunderkrankungen zu erleichtern. In wie weit objektivierende und quantifizierende Messverfahren in der klinischen Routine Eingang finden werden, erscheint angesichts der Komplexität der Systeme jedoch noch ungewiss. Unbestritten bleibt die enorme Bedeutung einer genauen klinischen Beobachtung und Untersuchung des Gangs für die Diagnostik und die Einschätzung von erwünschten und unerwünschten Wirkungen der Behandlung psychiatrischer Erkrankungen. Der geschulte klinische Blick des erfahrenen Psychiaters oder Nervenarztes und die gründliche neurologische Untersuchung bleiben auch bei der Beurteilung von Gangstörungen auf dem Feld der Psychiatrie unerlässlich.

\section{Gait disturbances in psychiatric disorders}

Gait disturbances are a common symptom of various psychiatric disorders. They can result in distinctive limitations of mobility and are of immense personal and socio-economic importance for the patient. Human gait is a complex automated process and requires undisturbed interaction of various brain functions. Several methods like gait analysis according to Brinkmann, videography, or ultrasonic and infra-red systems are used to objectively study gait disturbances. By means of these techniques a detailed investigation of gait disorders is possible. For the clinician it is important to detect dissociative gait disturbances in patients. Furthermore, gait disturbances can occur with affective disorders and schizophrenia. When gait is disturbed in schizophrenic patients, it is relevant to differentiate between symptoms of the disorder itself and extrapyramidal motor side-effects of antipsychotic drugs.

\section{Key Words}

gait disturbances - psychiatric disorders - diagnostic - schizophrenia

\section{Literatur}

1. Chouinard G, Ross-Chouinard A, Annable L, Jones BD. The Extrapyramidal Symptoms Rating Scale. Can J Neurol Sci 1980; 7: 233 244

2. Hayes MW, Shynti GB, Heldorf P, deMoore $G$, Morris JG. A Video Review of the Diagnosis of Psychogenetic Gait: Appendix and Commentary. Mov Disord 1999; 14: 914 921

3. Hennerici M, Bäzner H. Gangstörungen. Grundlagen und computergestützte Analyse. Berlin: Springer,2001: 1-14

4. Hirokawa S. Normal gait characteristics under temporal and distance constraints. J Biomed Eng 1989; 11: 449-456

5. Lemke MR, Broderick A, Zeitelberger M, Hartmann W. Motor Activity and Daily Variation of Symptom Intensity in Depressed Patients. Neuropsychobiology 1997; 36: 57-61

6. Lemke MR, Wendorff T, Mieth B, Buhl K, Linnemann M. Spatiotemporal gait patterns during over ground locomotion in major depression compared with healthy controls. J Psychiat Res 2000; 34: 277-283

7. Putzhammer A, Heindl B, Müller J, Broll $K$, Pfeiff L, Perfahl M, Hess L, Koch H. Dreidimensionale ultraschallgestützte Ganganalyse bei schizophrenen Patienten. Vergleich zwischen konventionellen Neuroleptika und Olanzapin. Psychiat Prax 2003; 30 (Suppl. 2): 110-114

8. Putzhammer A, Heindl B, Broll K, Pfeiff L, Perfahl M, Hajak G. Spatial and temporal parameter of gait disturbances in schizophrenic patients. Schizophr Res 2004; 69: 159-166

9. Putzhammer A, Perfahl M, Pfeiff L, Hajak G. Gait disturbances in patients with schizophrenia and adaptation to treadmill walking. Psychiatry Clin Neurosci 2005; 59: 303-310

10. Rubino FA. Gait Disorders. Neurologist 2002; 8: 254-262

11. Sloman L, Berridge M, Homatidis S, Hunter D, Duck T. Gait patterns of depressed Patients and normal subjects. Am J Psychiatry 1982; 139: 94-97

12. Wendorff T, Linnemann M, Lemke MR. Lokomotion und Depression. Klinik und Physiologie des Ganges bei Morbus Parkinson und affektiven Störungen. Fortschr Neurol Psychiat 2002; 70: 289-296

13. Whittle MW. Gait analysis. An introduction. Oxford: Butterworth-Heinemann, 1991

\section{Korrespondenzadressen:}

Maria Perfahl und

Dr. med. Albert Putzhammer

Klinik und Poliklinik für Psychiatrie,

Psychosomatik und Psychotherapie

der Universität Regensburg

Universitätsstraße 84

93053 Regensburg

Maria.Perfahl@medbo.de

Albert.Putzhammer@medbo.de 